\title{
La investigación sobre personas mayores y publicidad: análisis metodológico (1976-2012)
}

\author{
Irene RAMOS-SOLER \\ Universidad de Alicante \\ Irene.Ramos@ua.es \\ José F. MANCEBO-ARACIL \\ Universidad de Alicante \\ jf_mancebo@hotmail.com
}

Recibido: 05/11/2012

Aceptado: 23/01/2013

\section{Resumen}

Las personas mayores se han convertido en un segmento de mercado muy interesante debido a su peso económico y demográfico, siendo sus miembros grandes consumidores de los medios de comunicación. En este sentido y desde ámbito de la investigación académica en comunicación, el público senior se presenta como un tema de investigación oportuno y actual. El objetivo general de este trabajo es conocer cómo se ha procedido en la investigación científica en los estudios sobre personas mayores y publicidad, realizando para ello un análisis de contenido de los artículos publicadas durante el periodo 1976-2012. Los resultados de este estudio muestran la metodología y los temas preponderantes por décadas e identifican, entre otros aspectos, las variables de estudio más analizadas.

Palabras clave: Metodología; mayores; publicidad; análisis de contenido; revista académica

\section{The Investigation of Elderly People and Advertising: Methodological Analysis (1976-2012)}

\begin{abstract}
Older people have become a very interesting market segment due to its economic and demographic weight, its members being large consumers of media. In this sense and from academic research field of communication, the senior public comes as a appropriate research topic and current. The overall objective of this study was to determine the projection and the use given to each of the research methodologies in studies of elderly and advertising doing a content analysis of articles published during the period 1976-2012. The results of this study show the methodology and predominants themes for decades and identify, among other things, the study variables analyzed.
\end{abstract}

Keywords: Methodology; elderly population; advertising; content analysis, academic journal.

\section{Referencia normalizada}

RAMOS-SOLER, Irene y MANCEBO-ARACIL, José F. (2013): "La investigación sobre personas mayores y publicidad: análisis metodológico (1976-2012)". Estudios sobre el mensaje periodístico. Vol. 19. Núm. especial abril, págs.: 945-952. Madrid, Servicio de Publicaciones de la Universidad Complutense.

Sumario: 1. Introducción. 2. Metodología. 3. Resultados. 4. Conclusiones. 5. Referencias bibliográficas.

\section{Introducción}

En la actualidad, el perfil demográfico de la mayoría de los países desarrollados es el de una sociedad envejecida, al descenso de la tasa de natalidad hay que unir el hecho de que la esperanza de vida es cada vez mayor, vivimos más tiempo y en mejores condiciones físicas.

Las consecuencias sociales y económicas de este hecho se estudian por investigadores desde ámbitos diversos como la sociología, la economía, la demografía, la psicología, la medicina y la comunicación. 
En la literatura especializada se habla del impacto que el envejecimiento de la población tendrá tanto a nivel social como económico, y se estudian los nuevos escenarios y situaciones que se dibujan en los que las personas mayores ocuparán un papel central.

Gradualmente se han ido incorporando a la tercera edad nuevas generaciones de personas mayores que son muy diferentes de las que les precedieron. Los nuevos mayores han nacido y se han desarrollado en una sociedad de consumo, tienen un nivel económico y cultural más elevado que el de generaciones anteriores y se sienten activos y en forma.

Las personas mayores son un grupo heterogéneo, con estilos de vida, necesidades y procesos de envejecimiento diferentes y personales, lo que desafía de manera contundente los estereotipos y falsos mitos que han rodeado, hasta hace poco, a este grupo de población.

Sin embargo tal y como afirman Gómez-Calcerreda y Navarro (2009) en los medios de comunicación y en la publicidad todavía se recogen muchos aspectos de los mitos más extendidos de la vejez.

El público senior se han convertido en un segmento de mercado muy interesante debido a su peso económico, pero sobre todo demográfico, siendo sus miembros grandes consumidores de los medios de comunicación (Hudson, S., 2010; Westerhof et al., 2010; Sudbury, L. y Simcock, P.,2009; Kohlbacher y Herstatt, 2008; Furlong, 2007; Ramos, 2007; Thornhill y Martin, 2007).

En este sentido y desde ámbito de la investigación académica en comunicación, el público sénior se presenta como un tema de investigación oportuno y actual.

La investigación académica sobre las personas mayores y la publicidad es reciente en España existiendo una literatura más abundante y rica en el ámbito anglosajón (Ramos-Soler y Carretón, 2012). Sin embargo, llama la atención la escasez de estudios de contenido sobre metodologías de investigación en el ámbito de las personas mayores y la comunicación.

El presente trabajo se centra precisamente en este ámbito de trabajo, pretendiendo realizar una primera aportación en el ámbito de la investigación metodológica.

La elección de un método u otro dependen de la pregunta de investigación planteada y de su traducción en objetivos e hipótesis. Para Roca y Mensa (2009:12) "los investigadores seleccionan una metodología apropiada para obtener resultados y establecer inferencias". Investigar el pluralismo metodológico es también investigar los diversos modos de aproximación, descubrimiento y justificación en atención a la faceta o dimensión de la realidad social que se quiera estudiar (Beltran, 1985).

Estudiar la fundamentación teórica, metodológica y el estado actual de las investigaciones sobre este tema es una de las prioridades de este trabajo, siendo el objetivo principal conocer la proyección y el uso que se da a cada una de las metodologías de investigación científica en los estudios sobre personas mayores y publicidad. De esta manera, se pretende responder la siguiente pregunta de investigación: "¿Qué metodología es la más utilizada en los artículos publicados en el ámbito nacional y anglosajón en los últimos 30 años? Este objetivo general se aborda a través de los siguientes objetivos específicos: 
1. Conocer la frecuencia de publicación de artículos teóricos frente a los empíricos.

2. Establecer las metodologías y las técnicas cuantitativas y cualitativas más utilizadas.

3. Conocer las variables de estudio más analizadas.

Tal y como señalan Garcia y Berganza (2005:28) "las diferentes vías metodológicas de acceso a la realidad social configuran el marco epistemológico en que se plantean las investigaciones y donde se articulan las diversas técnicas de investigación social".

\section{Metodología}

Según West (2007) la investigación metodológica se focaliza en los métodos de investigación utilizados en un número de estudios sobre el mismo tema o la misma disciplina. De acuerdo con este autor realizamos en esta investigación un análisis de contenido de los artículos publicados durante el periodo 1976-2012 en revistas españolas y anglosajonas indexadas en las principales bases de datos ISI-Wok, Scopus, ESBCO y en el caso español In-Recs y Latindex. Para la búsqueda y selección de los artículos se utilizaron las siguientes palabras clave: personas mayores, publicidad, comunicación, tercera edad, público sénior, medios de comunicación, ancianos y envejecimiento.

Siguiendo la metodología realizada por Roca y Mensa (2009) se has estudiado doce variables agrupadas en cinco secciones. La primera sección dedicada a la descripción de la muestra, está formada por cinco variables "Década de publicación", "Temática de la revista", "País de la revista", "Número total de autores" y "Ámbito geográfico del estudio".

La segunda sección clasifica los estudios publicados en las diferentes revistas en función del tipo de estudio: "Teórico", artículos que parten de un marco teórico y permanecen en él sin contrastarlos con ningún aspecto práctico, y "Empírico" trabajos en los que las evidencias derivadas de la investigación adoptan la forma de algún tipo de datos (cualitativos o cuantitativos) y el investigador basa su trabajo en dichos datos.

La sección tercera contiene tres variables, la primera llamada "Investigación Empírica" en la que los datos se codifican como: investigación cualitativa, investigación cuantitativa o ambas. La segunda variable de esta tercera sección corresponde a la "investigación cualitativa" que clasifica los artículos publicados en: grupo de discusión, observación, entrevista, estudio de casos y delphi. La última variable corresponde a la "investigación cuantitativa" y se divide en: análisis de contenido, encuesta y experimentación.

En la sección quinta la variable "Edad" clasifica los artículos en función de la edad a partir de la cual consideran a una persona mayor o senior. Finalmente, en la sección quinta, se incluyen las variables "medios de comunicación" y "variables estudiadas". Las categorías utilizadas en esta última son: género, raza, importancia o magnitud, rol desempeñado, imagen social y carácter, imagen física, competencia física y mental, escenario, forma de vestir, público objetivo de la comunicación, producto anun- 
ciado, valores asociados, tamaño/tiempo del anuncio, intergeneracionalidad y estructura audiovisual-narrativa.

Para la codificación y el análisis estadístico de los datos recogidos se ha utilizado el programa SPSS.

\section{Resultados}

El primer artículo encontrado sobre publicidad y mayores data de los años setenta. Sin embargo, como podemos observar en la tabla siguiente, es a partir de 1980 cuando la regularidad en publicaciones científicas sobre este tema es más visible, con una cantidad de artículos muy parecida en las décadas posteriores hasta la actualidad.

Tabla 1. Década de publicación. Elaboración propia

\begin{tabular}{|l|c|c|c|}
\hline Década & Frecuencia & \% & \% Acumulado \\
\hline $1976-1979$ & 1 & 2,3 & 2,3 \\
\hline $1980-1989$ & 11 & 25,6 & 27,9 \\
\hline $1990-1999$ & 12 & 27,9 & 55,8 \\
\hline $2000-2009$ & 14 & 32,6 & 88,4 \\
\hline $2010-2020^{*}$ & 5 & 11,6 & 100,0 \\
\hline Total & 43 & 100,0 & \\
\hline
\end{tabular}

En cuanto a los medios revisados y su país de publicación, los resultados muestran que EEUU y Reino Unido son referentes en cuanto a número de publicaciones científicas, con el 61 y el $23 \%$ de las publicaciones localizadas para el presente estudio.

Se ha estudiado si existe relación entre el país de origen de las revistas analizadas y el ámbito geográfico de estudio de los artículos publicados. Para ello, se aplicó el Test Chi-Cuadrado, que contrasta la hipótesis nula de que dos variables o características son independientes, creando tablas de contingencias con cada una de las variables analizadas. Los resultados obtenidos muestran que el grado de significación del estadístico chi-cuadrado es inferior a 0,05 , por lo que se rechaza la hipótesis nula, luego no son independientes. De manera que el ámbito donde ha sido realizada la investigación influye en el país donde se ha publica el artículo.

Tabla 2. País de la Revista y Ámbito País del Estudio. Elaboración propia

\begin{tabular}{|l|c|c|c|c|c|c|c|c|}
\hline & \multicolumn{7}{|c|}{ Ámbito País del Estudio } & Total \\
\hline País revista & Canadá & EEUU & EEUU +Otros & España & Holanda & Japón & UK & \\
\hline Alemania & 0 & 0 & 0 & 1 & 0 & 2 & 0 & 3 \\
\hline Canadá & 1 & 0 & 0 & 0 & 0 & 0 & 0 & 1 \\
\hline EEUU & 1 & 22 & 3 & 0 & 0 & 0 & 0 & 26 \\
\hline España & 0 & 0 & 0 & 3 & 0 & 0 & 0 & 3 \\
\hline UK & 1 & 2 & 1 & 0 & 1 & 0 & 6 & 11 \\
\hline Total & $\mathbf{2}$ & $\mathbf{2 4}$ & $\mathbf{4}$ & $\mathbf{4}$ & $\mathbf{1}$ & $\mathbf{2}$ & $\mathbf{6}$ & $\mathbf{4 3}$ \\
\hline
\end{tabular}


Tabla 3. Pruebas de chi-cuadrado* (T2). Elaboración propia

\begin{tabular}{|l|c|c|c|}
\hline & Valor & gl & $\begin{array}{c}\text { Sig. asintótica } \\
\text { (bilateral) }\end{array}$ \\
\hline Chi-cuadrado de Pearson & $112,504(\mathrm{a})$ & 24 &, 000 \\
\hline Razón de verosimilitudes & 71,044 & 24 &, 000 \\
\hline N de casos válidos & 43 & & \\
\hline
\end{tabular}

En cuanto a la temática de las publicaciones de la muestra, se observa una distribución bastante homogénea, con un reparto similar y representativo en las tres categorías principales, tal y como muestra el gráfico siguiente.

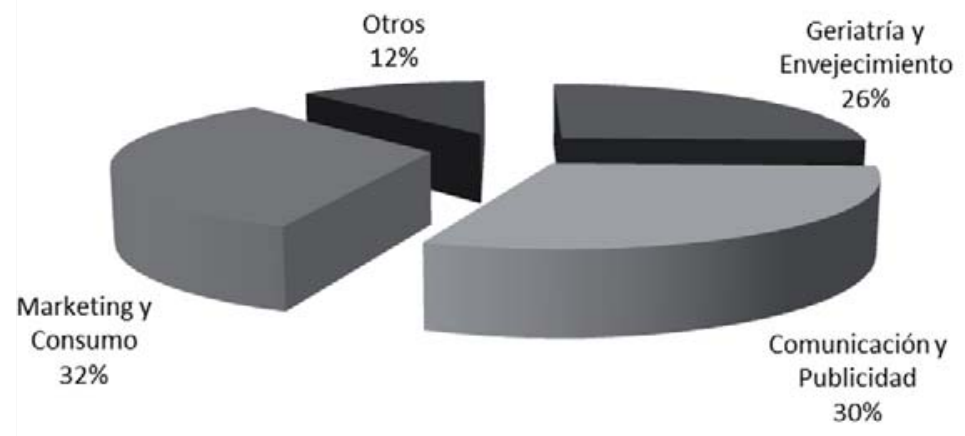

Gráfico 1. Temática de las revistas de la muestra. Fuente: Elaboración propia

La mayoría de los artículos publicados fueron firmados por uno (44\%)o dos autores $(26 \%)$. Aunque hay un número significativo de artículos firmados por tres y cuatro personas, bien es cierto que vemos como disminuye su frecuencia llegando, en casos muy puntuales, a ver artículos firmados por cinco y seis personas.)

Respecto a la frecuencia de publicación de artículos empíricos frente a teóricos, los resultados muestran que el $88,4 \%$ de los estudios son empíricos, estudiando mayoritariamente la representación de los mayores en la publicidad con una metodología cuantitativa. Si el artículo conllevaba una carga teórica importante, como una revisión bibliográfica exhaustiva pero mantenía algún estudio de carácter empírico (como un análisis de contenido de artículos anteriormente publicados, al modo del presente) se ha clasificado como empírico y se consideran ambas metodologías en un $4,7 \%$ de los casos.

En cuanto a las técnicas empleadas, se observa una gran preferencia por las técnicas cuantitativas, concretamente, el análisis de contenido y, en menor medida, la encuesta.

El medio de comunicación más analizado en los estudios sobre mayores y publicidad es el de las revistas (39\%), seguido muy de cerca por la televisión (37\%). La prensa apenas se trata en el $18 \%$ de los casos mientras que la radio tiene un carácter meramente anecdótico en los estudios realizados hasta la fecha, al igual que ocurre con los otros medios (Internet en sus facetas de Web 1.0 o 2.0).

Respecto a la edad a partir de la cual se considera a una persona mayor o sénior, destaca el escaso consenso que existe entre los investigadores que han publicado sobre 
este tema. A pesar de ello la mayoría de los estudios coinciden en considerar la edad de jubilación (65 años) sin embargo, a partir de los años 90 se observa comoempieza a bajar moderadamente la cifra hasta los 50 o más.

Las variables más utilizadas en los estudios de mayores y publicidad son, en al menos la mitad de los casos: la imagen social, el producto asociado a la comunicación, el rol y el género de la persona mayor. De otro lado, el tamaño y la estructura audiovisual narrativa del anuncio, la forma de vestir del mayor y el análisis del escenario donde aparece (interior, exterior, laboral, en casa, etc.) las variables que aparecen con una menor frecuencia.

Tabla 4. Variables estudiadas en los artículos sobre mayores y publicidad.

Elaboración propia.

\begin{tabular}{|l|c|}
\hline \multicolumn{1}{|c|}{ Pregunta } & \% Sí \\
\hline ¿Estudia la imagen social y carácter del mayor? & $\mathbf{6 7 , 4 4 \%}$ \\
\hline ¿Estudia el producto asociado a la comunicación con mayores? & $\mathbf{6 5 , 1 2 \%}$ \\
\hline ¿Estudia el rol desempeñado por el mayor? & $\mathbf{6 0 , 4 7 \%}$ \\
\hline ¿Estudia el género de la persona mayor estudiada? & $\mathbf{5 1 , 1 6 \%}$ \\
\hline ¿Estudia la magnitud o importancia del papel desempeñado por el mayor? & $44,19 \%$ \\
\hline ¿Estudia los valores asociados a la comunicación con mayores? & $41,86 \%$ \\
\hline ¿Estudia la imagen física del mayor? & $34,88 \%$ \\
\hline ¿Estudia la competencia física mental del mayor? & $30,23 \%$ \\
\hline ¿Estudia la raza/etnia? & $25,58 \%$ \\
\hline ¿Estudia el Target Group de la comunicación en la que aparece? & $23,26 \%$ \\
\hline ¿Estudia la presencia intergeneracional o no del mayor en la comunicación? & $20,93 \%$ \\
\hline ¿Estudia el escenario en el que aparece el mayor? & $\mathbf{1 8 , 6 0 \%}$ \\
\hline ¿Estudia la forma de vestir? & $\mathbf{1 1 , 6 3 \%}$ \\
\hline ¿Estudia de la estructura audiovisual-narrativa? & $\mathbf{9 , 3 0 \%}$ \\
\hline ¿Estudia el tamaño del anuncio en el medio (Prensa, Internet, Radio, TV)? & $\mathbf{4 , 6 5 \%}$ \\
\hline
\end{tabular}

\section{Conclusiones}

En este trabajo se han estudiado de forma empírica las metodologías empleadas en la investigación académica sobre personas mayores y la publicidad cuyos resultados se han publicado en revistas académicas durante los últimos cuarenta años.

Salvando algunos esfuerzos teóricos muy interesantes (Carrigan y Szmigin, 1999 y 2000) o con visión trasnacional (Bing Zhang et Al., 2006), las revistas académicas han publicado principalmente trabajos empíricos de carácter cuantitativo, siendo el análisis de contenido la principal técnica utilizada en el ámbito que nos ocupa durante el periodo estudiado.

Llama la atención que no se haya realizado ningún análisis de contenido centrado en la radio, pese a ser uno de los medios de comunicación más cercanos y utilizados 
por el público sénior. Tampoco son muy frecuentes los estudios centrados en la prensa generalista. No se consideran teorías de la imagen, como la del framing para este tipo de estudios, aunque algunos estudios teóricos se esfuerzan en presentar recomendaciones en un intento de estudiar las tendencias actuales (como en Hudson, 2010).

El análisis de los diversos modos de aproximación a la realidad estudiada nos muestra que se ha trabajado fundamentalmente con medios en los que la imagen publicitaria tiene un papel fundamental como es el caso de la televisión y las revistas, estudiándose fundamentalmente variables que analizan esa imagen desde un punto de vista fisico, social y de comportamiento. Si la elección de un método u otro dependen de la pregunta de investigación planteada y de su traducción en objetivos e hipótesis, se puede concluir que el principal objeto de estudio durante los útimos años ha sido la presencia o no de las personas mayores en la publicidad y el estudio o análisis de su imagen.

\section{Referencias bibliográficas}

BELTRAN, Miguel, (1985): "Cinco vías de acceso a la realidad social", en REIS, n²9, enero-marzo, pp.7-42. España.

BING ZHANG, Yan; HARWOOD, Jake; WILLIAMS, Angie; YLÄNNE-MCEWEN, Virpi, WADLEIGH, Paul Mark; THIMM, Caja, (2006): "The Portrayal of Older Adults in Advertising: A Cross-National Review", en Journal of Language and Social Psychology, Vol.25, n 3, pp. 264-282. USA.

CARRIGAN, Marylyn; SZMIGIN, Isabelle, (1999): "The portrayal of older characters in magazine advertising", en Journal of Marketing Practice: Applied Marketing Science, MBC University Press, 5, 6/7/8, pp. 248-261. U.K.

CARRIGAN, Marylyn; SZMIGIN, Isabelle, (2000): "Advertising in an Ageing society, en Ageing and Society”, Cambridge University Press, 20, pp. 217-233. U.K.

CARRIGAN, Marylyn; SZMIGIN, Isabelle (2001): "Learning to love the older consumer. Journal of Consumer Behaviour", I (1), 22-34. U.K.

FURLONG, Mary (2007): Turning silver into gold. New Jersey (USA), Press Financial Times.

GARCÍA GALERA, Mª Carmen; BERGANZA CONDE, Ma Rosa (2005): “El método científico aplicado a la investigación en Comunicación Mediática". En BERGANZA CONDE, María Rosa y RUIZ SAN ROMAN, J. A.: Investigar en Comunicación (págs. 19-42). Madrid, McGrawHill.

GÓMEZ-CALCERREDA, Juan Luis y NAVARRO CORTÉS, Nuria (2009): "Los mitos y estereotipos sobre la vejez en los medios de comunicación y la importancia de la educación intergeneracional". Actas de Congresos en: http://www.imsersomayores.csic.es/documentos/documentos/fuentes-mitos-01.pdf

HUDSON, Simon (2010): "Wooing zoomers: marketing to the mature traveler". Marketing Intelligence and Planing , 444-461. EEUU.

KOHLBACHER, Florian y HERSTATT, Cornelius (2008): The silver market phenomenon. Berlin, Springer. Berlin. 
RAMOS-SOLER, Irene (2007): El estilo de vida de los mayores y la publicidad. Barcelona, Fundación "La Caixa".

RAMOS-SOLER, Irene y CARRETÓN BALLESTER, Ma Carmen (2012): "Presencia y representación de las personas mayores en la publicidad televisiva: El caso español”, en Revista Española de Geriatría y Gerontología. Vol. 47, n 2, pp.55-61.

ROCA, David; MENSA, Marta, (2009): "Metodologías utilizadas en las investigaciones de creatividad publicitaria (1965-2007)", en Comunicación y Sociedad. Vol XXII, $n^{\circ} 1$, pp. 7-34.

SUDBURY, Linn Y SIMCOCK, Peter (2009): "Understanding older consumers through cognitive age and the list of values: A U.K.-based perspective", en Psychology and Marketing, vol.26, 1, pp. 22-38. U.K.

THORNHILL, Matt; MARTIN, Jonh (2007): Boomer Consumer. Virginia (USA), Great Falls: A Linx Book..

WEST, Douglas (2007): "Directions in marketing communications research. An analysis of the International Journal of Advertising". International Journal of Advertising, 543-554. U.K.

WESTERHOF, Gerben J.; HARINK, Karolien; VAN SELM, Martine; STRICK, Madelijn; VAN BAAREN, Rick (2010): "Filling a missing link: the influence of portrayals of older characters in television commercials on the memory perfomance of older adults". Ageing and Society (30), 897-912. U.K.

\section{Irene RAMOS-SOLER}

Universidad de Alicante

Facultad de Ciencias Económicas y Empresariales. Dpto. Comunicación y Psicología Social

Profesora contratada doctora. Directora del Grupo de Investigación “Comunicación y Públicos específicos"

Irene.Ramos@ua.es

\section{Jose F. MANCEBO-ARACIL}

Universidad de Alicante. Miembro del Grupo de Investigación “Comunicación y Públicos específicos".

Universidad Miguel Hernández. Profesor Asociado en IMEP (Instituto Mediterráneo de Estudios de Protocolo)

jf_mancebo@hotmail.com 